\title{
Laparoscopic ovarian reconstruction without suturing after cystectomy for endometrioma
}

\author{
P. G. Paul • Harneet Kaur • Dhivya Narasimhan • \\ Gaurav Chopade • Dimple Kandhari
}

Received: 22 February 2014 / Accepted: 4 June 2014 / Published online: 24 June 2014

(C) Springer-Verlag Berlin Heidelberg 2014

\begin{abstract}
The primary aim of this study is to evaluate the technique of ovarian reconstruction without suturing after laparoscopic cystectomy of endometrioma. The secondary aim is to find the pregnancy rate following this technique. The study is a prospective observational study (Canadian Task Force classification II-3). The interventions used in the study are laparoscopic ovarian cystectomy and reconstruction without suturing. Laparoscopic ovarian cystectomy was performed in 240 patients between May 2007 and April 2012 of which 182 consecutive patients who met the selection criteria were enrolled in the study. Intraoperatively, the cyst wall is completely enucleated. Ovarian tissue is kept apposed together with a bowel grasper for $5 \mathrm{~min}$ to reconstruct the ovary. No sutures are used for approximation of ovarian edges. The median (range) operating time for cystectomy and reconstruction was $22 \mathrm{~min}$ (15-75), and estimated blood loss was $50 \mathrm{ml}$ (30-200). The ovarian reconstruction was good in $84.6 \%$ of the cases, average in $10 \%$ and poor in $5.4 \%$ of the patients. Postoperative scan on day 1 showed pelvic collection (blood) in five cases $(20-50 \mathrm{ml}) .9 .89 \%$ had intraovarian haematoma of 2-3 cm which resolved spontaneously. All patients were followed at 1 month and pregnancy rate was calculated after a
\end{abstract}

\author{
P. G. Paul $(\bowtie) \cdot$ H. Kaur • D. Narasimhan · G. Chopade • \\ D. Kandhari \\ Centre for Advanced Endoscopy and Infertility Treatment, Paul's \\ Hospital, Vattekkattu road, Kaloor, Cochin, Kerala 682 017, India \\ e-mail: drpaulpg@gmail.com \\ H. Kaur \\ e-mail: harneet.dmc@gmail.com \\ D. Narasimhan \\ e-mail: drzesto6666@yahoo.com \\ G. Chopade \\ e-mail: hschopade@rediffmail.com \\ D. Kandhari \\ e-mail: dimple.kandhari@yahoo.co.in
}

minimum follow up of 12 months. Pregnancy rate was $50.7 \%$ (33 patients) in our study. Approximation of ovarian surface for ovarian reconstruction was associated with shorter operating times, good morphological ovarian reconstruction and comparable pregnancy outcome. This technique requires further well-designed randomized controlled trials.

Keywords Laparoscopic cystectomy · Endometrioma · Ovarian reconstruction

\section{Introduction}

Endometriotic cysts are among the most common ovarian cysts encountered during surgery [1]. Endometriomas can cause pelvic pain, infertility and dyspareunia, and the most preferred treatment is surgical [2]. Various laparoscopic techniques have been described for the treatment of ovarian endometriomas: cyst wall laser vaporization preceded or not by medical therapy, drainage and bipolar coagulation of the cyst wall and stripping of the cyst wall [3-5]. Laparoscopic stripping is the preferred and safer technique [6]. After stripping of the cyst wall, bleeding from the ovarian wound is controlled by bipolar coagulation, suturing, or tissue sealants [7, 8]. Each of these is associated with its own advantages and disadvantages.

We believe that the ovaries should be reconstructed after cystectomy for better functional outcome and reduced postoperative adhesions. We have been reconstructing the ovaries by approximating the ovarian edges with a bowel grasper for few minutes. This technique used minimal diathermy and avoided suturing, thus achieving shorter operating times without requiring endosuturing expertise. Without washing of the residual blood, we keep the adjacent surface of the ovary approximated with pressure for a few minutes and this helps in the reconstruction. The primary aim of this study is to evaluate the technique for ovarian reconstruction after 
Fig. 1 Preoperative ultrasoundendometrioma

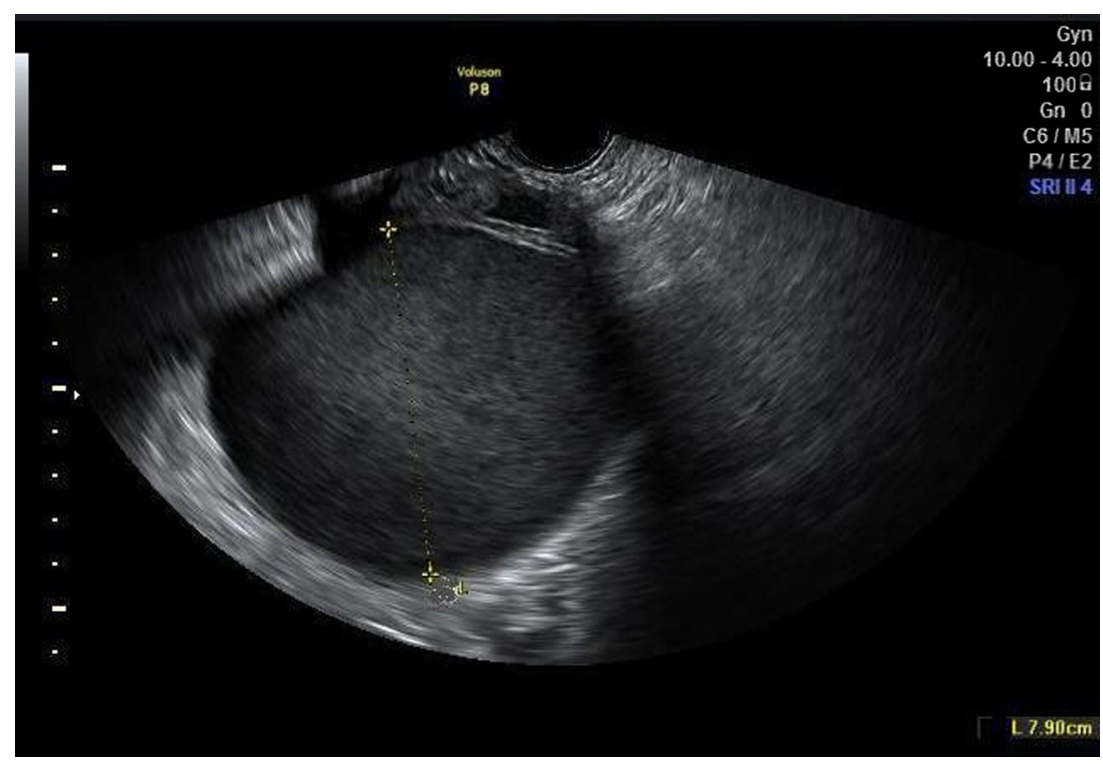

laparoscopic cystectomy of the endometrioma. The secondary aim is to find the pregnancy rate following this technique.

\section{Materials and methods}

This is a prospective observational study done at Paul's Hospital. A total of 240 patients underwent laparoscopic cystectomy for endometriosis between May 2007 and April 2012 at Paul's Hospital. Of these, 182 consecutive patients with endometriotic cyst $>3 \mathrm{~cm}$ in size were included in the study.

The exclusion criteria are the following:

1. Preoperative clinical diagnosis of non-endometriotic cyst

2. Severely distorted pelvic anatomy at surgery (i.e., large size uterine fibroids, severe adhesions, congenital abnormalities) which required additional surgical procedure with consequent increase of operating times and possible conversion to laparotomy

3. Previous surgery for endometriosis

4. Patients treated with gonadotropin-releasing hormone $(\mathrm{GnRH})$ analogues in the past 6 months

The institutional ethical committee of Paul's Hospital approved the data collection, aggregation, identification and analysis for this study. Informed consent is obtained from all patients. Data regarding patient characteristics like age, body mass index, parity, previous surgeries and intraoperative details like duration of surgery, complications, estimated blood loss, duration of hospital stay and postoperative events are evaluated. The outcome is evaluated as complete reconstruction, complications, symptomatic relief and pregnancy rate. Patients were excluded from data analysis if endometriosis was not confirmed at histopathology.

Fig. 2 Left endometrioma of $8 \mathrm{~cm}$

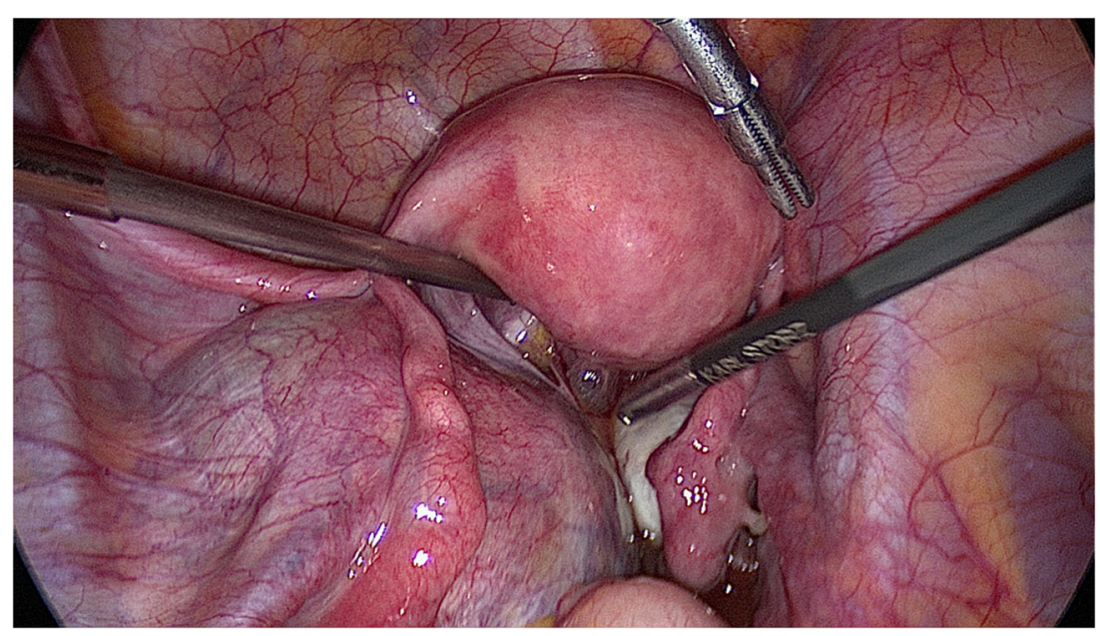


Fig. 3 Controlled tear on the edges of the cyst by traction with two graspers

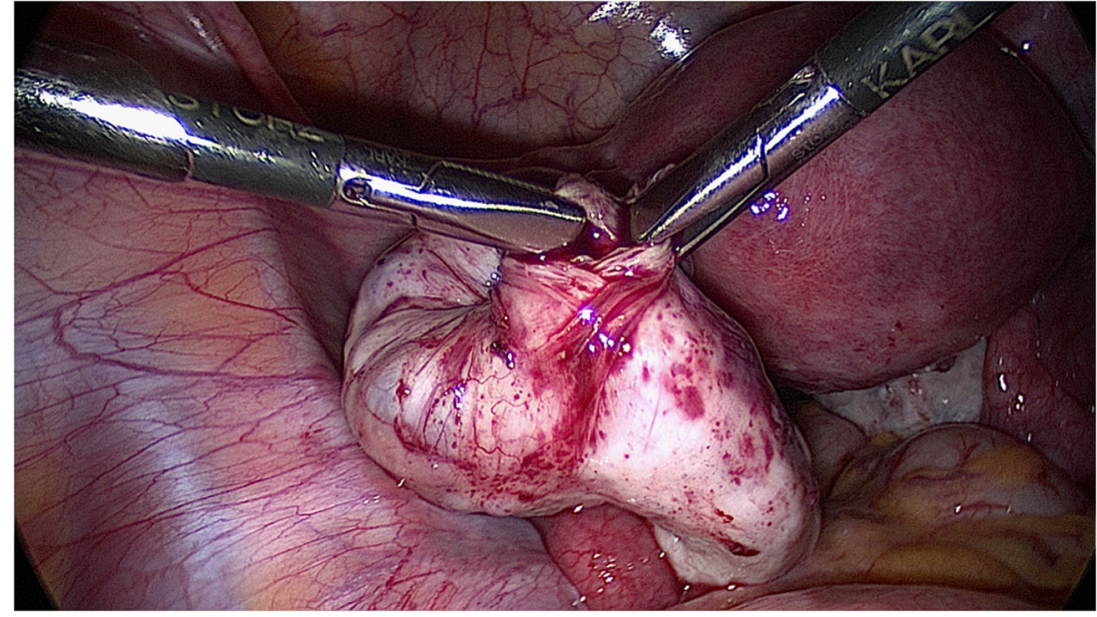

\section{Patient evaluation}

All patients are submitted to a detailed history regarding severity of abdominal pain, dysmenorrhoea, dyspareunia, bowel symptoms, previous abdominopelvic infections and surgeries. Clinical examination and transvaginal ultrasonography are done by the operating surgeon prior to surgery (Fig. 1). In celibate patients, transabdominal ultrasound is done. MRI is not routinely done.

Patients are admitted to the hospital on the day of surgery and kept nil per orally for $6 \mathrm{~h}$ prior to surgery. Bowel preparation is performed using sodium phosphate solution enema. Antibiotic prophylaxis is given at the time of induction of anaesthesia. Procedures are performed under general anaesthesia. All surgical procedures are carried out by the first author.

The patients are discharged on postoperative day 1 of surgery, and ultrasound examination is done for any pelvic or intraovarian haematoma before discharge (Fig. 6). All patients are followed up at 1 and 12 months and evaluated for any symptoms, and pregnancy rate was calculated. Postal questionnaires are sent to all patients, and telephonic enquiries are made at the end of the study. Minimum follow-up period is 1 year.

\section{Surgical technique}

Pneumoperitoneum is created using Veress needle at the umbilicus or at the Palmer's point. Peritoneal entry is done by direct trocar insertion or visual entry technique using Ternamian EndoTIP (Karl Storz, Tuttlingen) at the umbilicus or supraumbilical in patients with large abdominal masses. Abdominopelvic inspection is done for omental and bowel adhesions and to confirm the preoperative diagnosis (Fig. 2). Three accessory port techniques are used, two ports in the lower quadrants lateral to the inferior epigastric artery and the third port in the suprapubic area. Endometriosis is staged according to the revised American Fertility Society (rAFS) classification [9].

Since 2 years, we have been using dilute vasopressin for generalized vasoconstriction in the pelvis which helps in achieving initial haemostatic dissection of the adnexa and
Fig. 4 Separation of cyst wall from the ovarian tissue

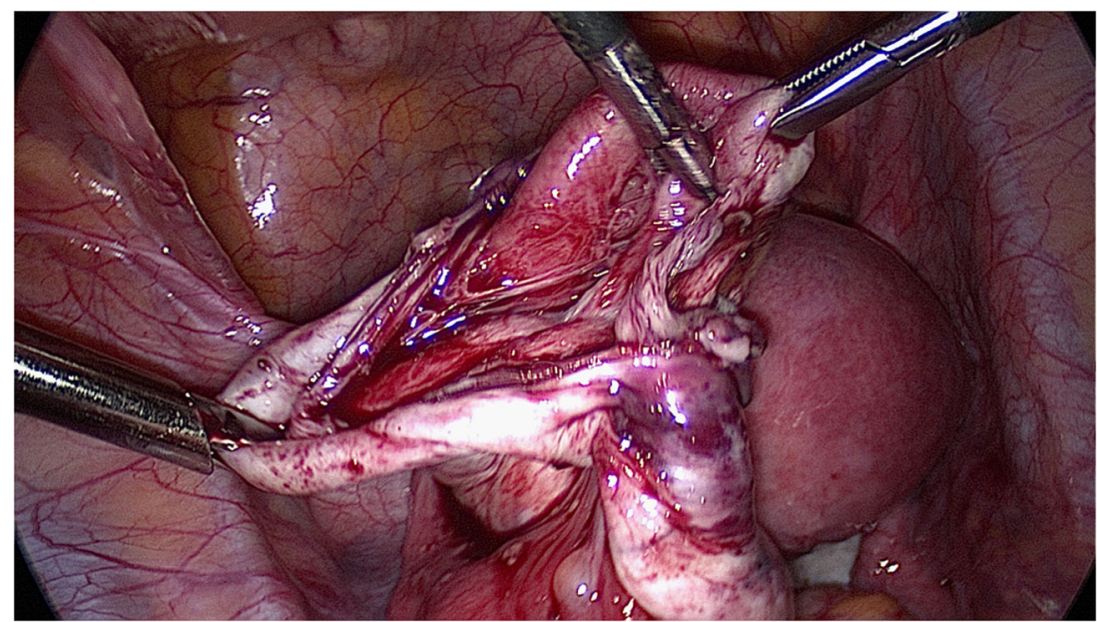


Fig. 5 Approximation of the edges with bowel grasper

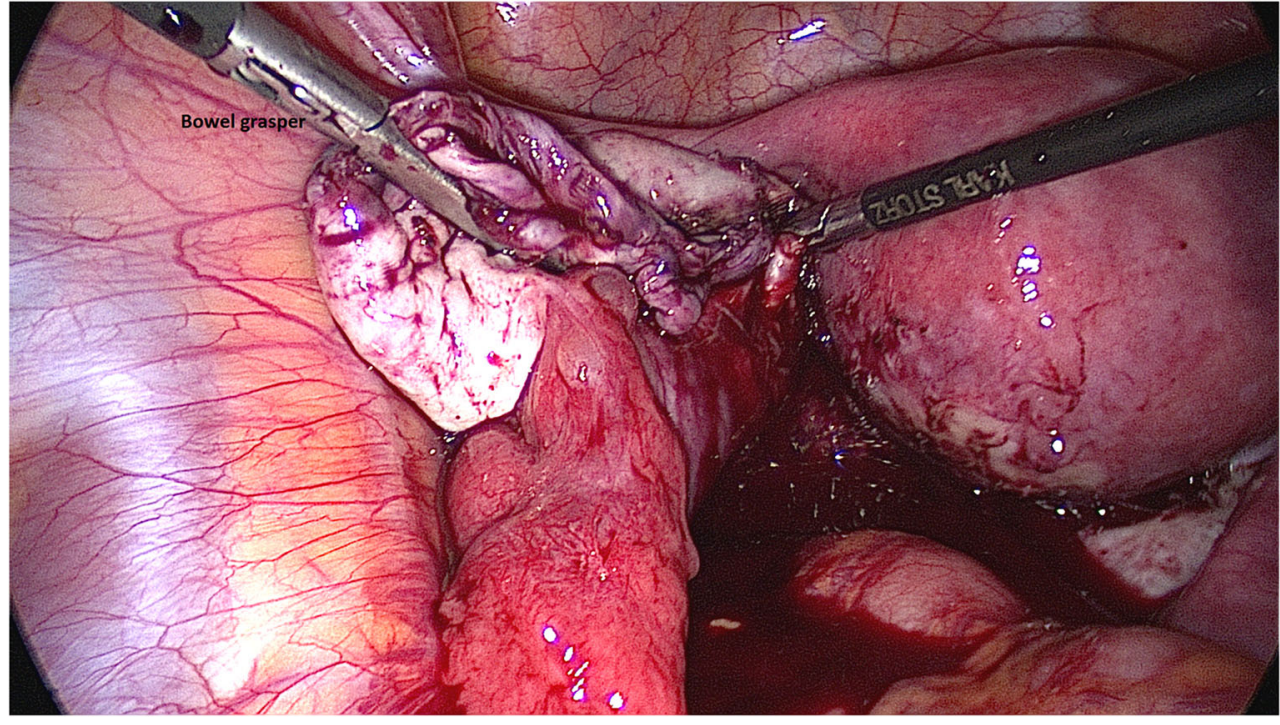

rectosigmoid. Twenty units of vasopressin is diluted in $100 \mathrm{ml}$ of normal saline, and 40 to $60 \mathrm{ml}$ of this solution is injected into the myometrium till the uterus is blanched. After anteverting the uterus with a Spackman cannula, salpingoovariolysis is done with sharp dissection. All fleshy adhesions from the uterus and pouch of Douglas are excised. All superficial endometriosis implants are coagulated, and deep ones are excised.

The ovary with small endometrioma is mobilized from the pelvic ovarian fossa, and when the chocolate material invariably leaks out, the leaked out contents are aspirated, followed by complete irrigation and aspiration of the cyst. For large endometrioma, we puncture the cyst with trocar and aspirate the chocolate material. We make a controlled tear of $1 \mathrm{~cm}$ on the edge of the cyst by traction with two graspers, rather than using sharp cutting (Fig. 3). This step separates the cyst wall from the ovarian tissue. After identification of the cleavage plane, the cyst wall is enucleated through traction exerted in opposite directions with two grasping forceps. The cyst wall is held with a toothed grasper, and ovarian tissue is held with an atraumatic grasper. Traction and countertraction are done to separate the cyst from the ovarian tissue (Fig. 4). Complete removal of the cyst wall is done. Enucleation is done gently near the ovarian ligament and hilum to avoid bleeding and injury. Any residual endometriosis on the edges of the ovary is excised. At the end of the procedure, only the active bleeding vessel is controlled with bipolar coagulation. No sutures are used for approximation of the ovarian edges. The ovarian edges are held together with three graspers, and by trial and error, the best symmetrical approximation of the edges is achieved. Ipsilateral grasper is replaced with the bowel grasper to achieve the maximal approximation of the ovarian edges. The suprapubic grasper is released once the bowel grasper is in proper position. The specimen is removed through the 10$\mathrm{mm}$ primary trocar after changing the camera to a $5-\mathrm{mm}$ telescope introduced through the suprapubic port. Large cysts were removed with an endobag after enlarging the primary port. The vaginal assistant holding the bowel grasper keeps
Fig. 6 Large ovarian endometrioma $(19 \times 6 \mathrm{~cm})$

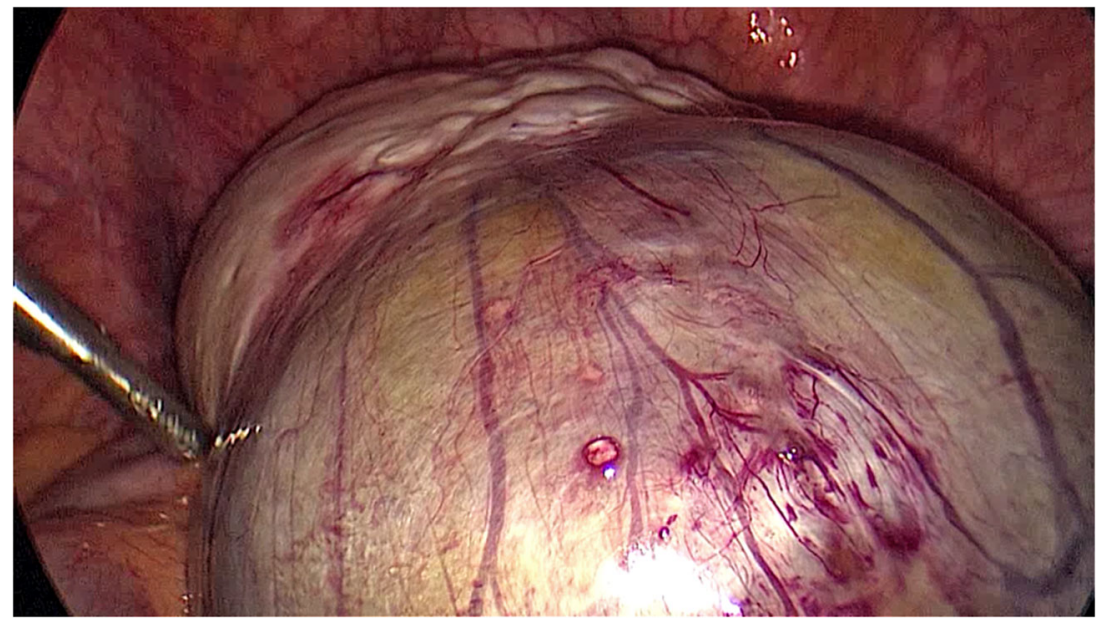


Fig. 7 Large ovarian endometrioma reconstructed after cystectomy with bowel graspers

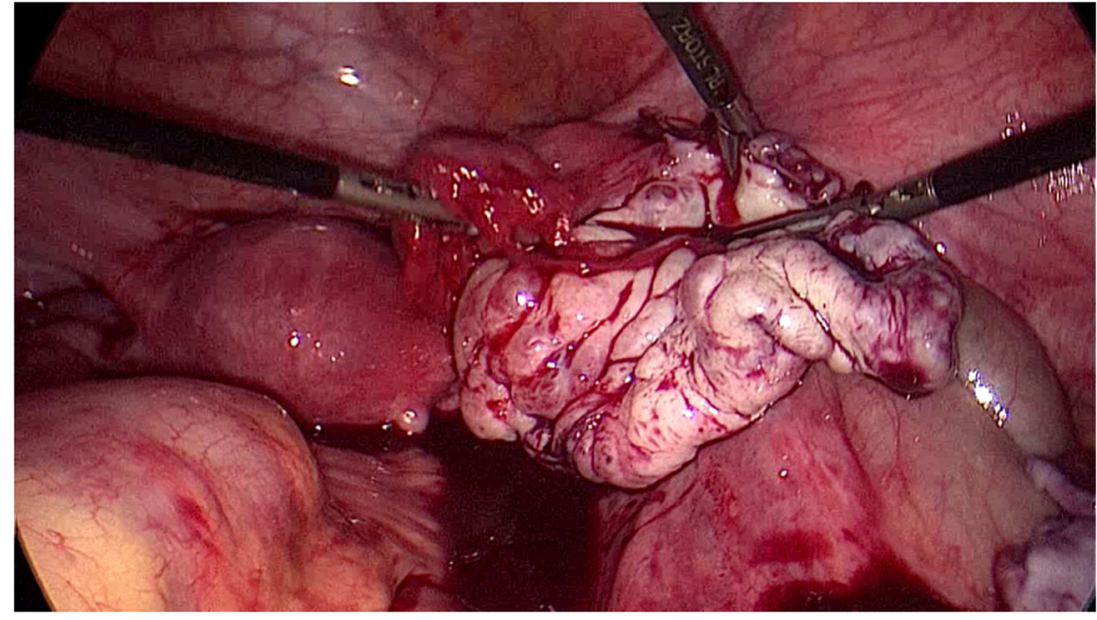

the forearm rested on the corresponding thigh of the patient and is not involved in specimen retrieval. The edges are hence approximated, and the tissue is held together for $5 \mathrm{~min}$ (Fig. 5). Trimming of the ovarian edges is rarely needed and done only to excise endometrial implants. For very large cysts, the bowel grasper is applied more towards the edges and rotated laterally to get a better approximation of the inner wall of the ovary (Figs. 6 and 7). The uterus can be deviated laterally with the manipulator to compress the ovary against the pelvic side wall. Rarely, ovarian reconstruction cannot be achieved due to a persistent bleeder from the cystectomy site. In such a situation, the ovary has to be reopened and the offending bleeding is controlled, and the aforementioned steps are repeated to achieve ovarian reconstruction. The ipsilateral bowel grasper and the contralateral graspers are released, and the ovary is inspected whether it is fully reconstructed (Fig. 8). The morphological appearance of the ovary is described as good reconstruction if the surface apposition was $>75 \%$, average if the surface apposition was $50-75 \%$ and poor if the surface apposition was $<50 \%$.

\section{Findings}

Laparoscopic ovarian cystectomy was performed in 240 patients with endometriosis between May 2007 and April 2012. Of these patients, 33 patients had previous surgery for endometriosis, 7 patients were previously treated with GnRH analogues, 15 patients had severely distorted pelvic anatomy at surgery requiring additional surgical procedures and 5 patients were detected with non-endometriotic cysts and hence excluded from the study. Hence, 182 patients were enrolled in the study. Patients' data are shown in Table 1 . The mean \pm standard deviation (SD) age of patients was $30.37 \pm 5.7$ years; body mass index was $23.8 \pm 4.2$. Fifty-nine patients had previous abdominal surgery (laparotomy, laparoscopy for indications other than endometriosis and did not have severely distorted pelvic anatomy). Out of the total, 16 patients were celibate, 124 were nulliparous and 42 were parous, of which 20 had previous caesarean section. The presenting complaints of patients were dysmenorrhoea in 68 patients and abdominal pain in 47. In 101 patients presented with infertility (duration

Fig. 8 Ovary after reconstruction

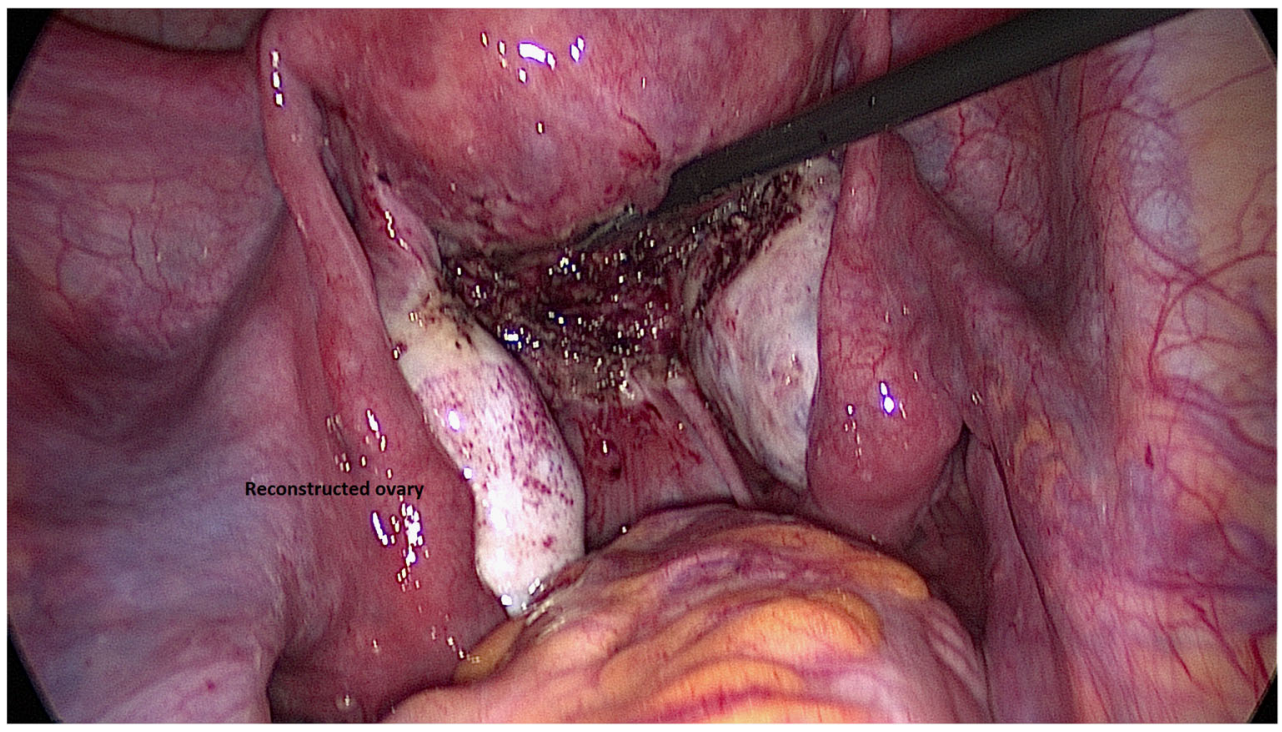


Table 1 Demographic characteristics/patient details

\begin{tabular}{ll}
\hline Variable & No. of cases \\
\hline Age & $30.37 \pm 5.7$ \\
BMI & $23.8 \pm 4.2$ \\
Presenting complaints & \\
Dysmenorrhoea & 68 \\
Pain abdomen & 47 \\
Primary in fertility & 72 \\
Secondary infertility & 29 \\
Dyspareunia & 2 \\
Deliveries & \\
Vaginal & 20 \\
Caesarean section & 20 \\
Parity & \\
Celibate & 16 \\
0 & 124 \\
1 & 35 \\
$\geq 2$ & 7 \\
Previous surgeries & 59 \\
1 & 18 \\
2 & 41 \\
\hline
\end{tabular}

of infertility ranging from 1 to 5 years), out of these patients, 31 had fibroid uterus and 5 had adenomyosis of the uterus with endometriosis and hence excluded from the pregnancy rate calculation to avoid multiple variables.

Preoperative sonography findings are tabulated in Table 2. The size and localization of endometriomas were documented.

Intraoperative findings and type of surgery are mentioned in Table 3. Seventy-five patients had bilateral cystectomy, and five patients who were not desirous of future fertility and had severe pelvic pain underwent unilateral adnexectomy of the relatively severely affected ovary and cystectomy of the remaining ovary. The median (range) operating time for cystectomy and reconstruction was 22 min (15-75) (from the beginning of cystectomy to removal of bowel grasper), and the estimated blood loss was $50 \mathrm{ml}(30-200)$. Since cystectomy was done as the last step, the suction aspirate was measured separately to evaluate the blood loss. Ovary

Table 2 Preoperative ultrasound findings

\begin{tabular}{llll}
\hline Size & Localization & & \\
\cline { 2 - 4 } & Right adnexa & Left adnexa & Bilateral \\
\hline $1-3 \mathrm{~cm}$ & 18 & 13 & 40 \\
$3-7 \mathrm{~cm}$ & 23 & 28 & 42 \\
$>7 \mathrm{~cm}$ & 5 & 7 & 6 \\
Total & 46 & 48 & 88 \\
\hline
\end{tabular}

Table 3 Operative details

\begin{tabular}{ll}
\hline rAFS score median ( range) 24 (16-94) & No. of cases \\
\hline Stage III & 68 \\
Stage IV & 114 \\
Type of surgery & \\
Unilateral cystectomy & 66 \\
$\quad$ Right & 32 \\
$\quad$ Left & 34 \\
Bilateral cystectomy & 75 \\
Cystectomy with unilateral adnexectomy & 5 \\
Unilateral cystectomy with myomectomy & 21 \\
Unilateral cystectomy with hysteroscopic & 2 \\
polypectomy & 10 \\
Bilateral cystectomy with myomectomy & 2 \\
Bilateral cystectomy with hysteroscopic & 1 \\
polypectomy & \\
Bilateral cystectomy with hysteroscopic & $22 \mathrm{~min}(15-75)$ \\
myomectomy & $50 \mathrm{ml}(30-200)$ \\
Duration of cystectomy and reconstruction & $154(84.6 \%)$ \\
Blood loss & $18 \%(10 \%)$ \\
Morphological appearance of reconstructed ovary & \\
Good ( $>75 \%$ reconstructed) & \\
Average (50-75\%) & \\
Poor (<50 \%) & \\
\hline
\end{tabular}

rAFS revised American Fertility Society

reconstruction was good in $84.6 \%$ of the cases, average in $10 \%$ and poor in $5.4 \%$ of the patients (Table 3 ).

Suturing of the ovarian edges was required in two patients as the ovarian tissue had irregular edges, and reconstruction was not possible otherwise. None of the patients required conversion to laparotomy. No intraoperative or postoperative complication occurred. Length of postoperative stay was 1 day (1-4).

Postoperative scan reports are tabulated in Table 4. Postoperative ultrasonography on day 1 showed pelvic collection (blood) in five cases (20-50 ml) (Fig. 9). Ninety percent of the patients had no intraovarian haematoma. Haematoma in the ovary greater than $2 \mathrm{~cm}$ was seen in 18 cases $(2-3 \mathrm{~cm})$. None

Table 4 Postoperative ultrasound findings

\begin{tabular}{ll}
\hline Ultrasound - size of the ovary & No. of cases \\
\hline $3-6 \mathrm{~cm}$ & $160(87.9 \%)$ \\
$>6 \mathrm{~cm}$ & $22(12.1 \%)$ \\
Intraovarian haematoma & \\
$<2 \mathrm{~cm}$ & $164(90.10 \%)$ \\
$2-3 \mathrm{~cm}$ & $18(9.89 \%)$ \\
Pelvic collection & \\
$<20 \mathrm{ml}$ & $177(97.25 \%)$ \\
$20-50 \mathrm{ml}$ & $5(2.75 \%)$ \\
\hline
\end{tabular}


Fig. 9 Postoperative day 1 ultrasound showing the reconstructed ovary

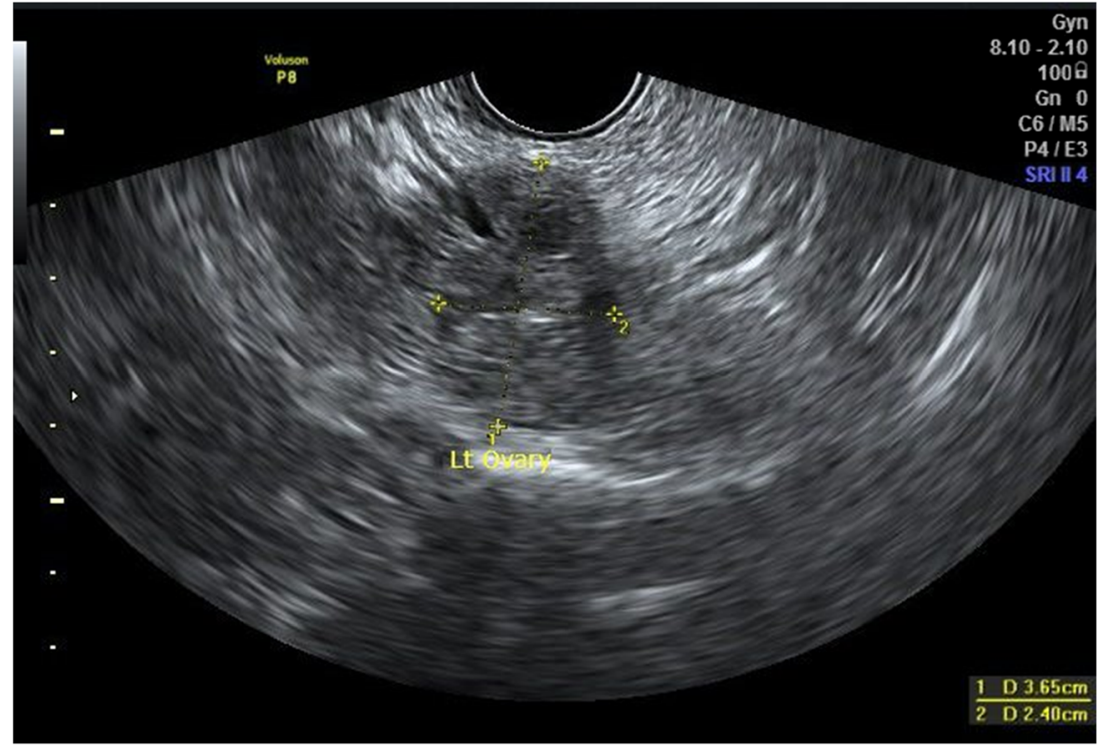

of these patients required any intervention and haematoma resolved on serial ultrasound scans.

Of the 65 patients desiring fertility, $21 \%$ of male partners had moderate oligoasthenozoospermia and $8 \%$ had severe oligoasthenozoospermia. Thirty-three patients $(50.7 \%)$ conceived and 22 conceived spontaneously and 11 after assisted reproductive technology (ART).

\section{Discussion}

In recent years, laparoscopy has become the gold standard for the treatment of ovarian endometriotic cysts $[10,11]$. Laparoscopic stripping is the most preferred procedure [6]. Good haemostasis is important after laparoscopic stripping of the cyst wall, and techniques like bipolar coagulation, sutures, fibrin sealants and direct pressure are used [8]. Bipolar coagulation of the ovarian stroma is effective but damages the healthy ovarian tissue. When the ovarian capsule is left open after bipolar coagulation, it is associated with more adhesions as compared to the reconstruction of the ovary [12]. In contrast, sutures are useful but also cause additional damage to the healthy tissue [13]. Though suturing technique has been shown to cause less adhesions as compared to bipolar coagulation by various studies, sutures cause more postoperative adhesions as seen in the myomectomy studies and suturing is technically more demanding [7, 14]. Thus, avoiding suturing will be a simpler technique for the reconstruction. In this study, we used compression with bowel grasper for ovarian reconstruction after laparoscopic cystectomy of endometrioma.

Our technique of laparoscopic stripping is similar to other studies, except that vasopressin is injected on the uterus rather than on the endometrioma $[6,15]$. On injecting vasopressin on the uterus, we get a generalized vasoconstriction of the pelvis which helps in maintaining a clear view without much bleeding during adhesiolysis.

With approximation of the adjacent surfaces of the ovary using the bowel graspers, the ovaries maintained its shape after removal and this is possibly due to the fibrin formed from the oozing ovarian surface. The empirical usage of $5 \mathrm{~min}$ correlates with the average clotting time of blood. Though the fibrinogen concentration of sealants is $15-25$ times higher than the circulating plasma, the endogenous fibrin was sufficient to stick the tissues in our study. Tissue sealants like FloSeal (gelantine thrombin matrix) sealant have been used to control bleeding following the ovarian stripping, but patients had postoperative pelvic pain with its use [8]. Fibrin sealants have been used in various urological procedures for controlling bleeding [16]. Fibrin glue has been used after laparoscopic excision of large endometriomas by Takeuchi et al. and had reduced postoperative adhesions as compared to the use of bipolar [12].

In our study, the ovarian reconstruction was good in $84.6 \%$, average in $10 \%$ and poor in $5.4 \%$ of the patients. Suturing of the ovarian edges was required in two patients only. We could not find any study describing the morphological appearance of the ovary after laparoscopic cystectomy.

Table 5 Pregnancy rate

\begin{tabular}{ll}
\hline Outcome & No. of cases \\
\hline Pregnancy & $33(50.7 \%)$ \\
Spontaneous conception & $22(66.6 \%)$ \\
Assisted reproductive technology (ART) & $11(33.2 \%)$ \\
Abortions & $5(15.1)$ \\
Term deliveries & $28(84.9 \%)$ \\
\hline
\end{tabular}


In our study, postoperative scan on day 1 showed pelvic fluid collection in five cases $(20-50 \mathrm{ml})$. In 18 cases, haematoma in the ovary was greater than $2 \mathrm{~cm}(2-3 \mathrm{~cm})$. Ninety percent of the patients had no intraovarian haematoma. None of these patients had evidence of infection and did not require any intervention. The pelvic collection and intraovarian haematoma resolved on serial ultrasound scans. In a case report by Ebert et al. using FloSeal for intraovarian haemostasis following cystectomy, day 2 ultrasound revealed a residuum of $10 \mathrm{~mm}$ within the ovary and residual fluid of less than $5 \mathrm{ml}$ in cul-de-sac [8]. Hence, the failure rate in our technique considering both morphological appearance $(5.4 \%)$ and postoperative ovarian haematoma $>2 \mathrm{~cm}(10 \%)$ was $15.4 \%$.

Pregnancy rate in our study was $50.7 \%$ (33 patients). Twenty-two patients had spontaneous pregnancy and 11 after ART (Table 5). Out of the 33 patients who conceived, 28 delivered and 5 had abortions. Vercellini et al. observed an overall crude pregnancy rate of $41 \%$ after primary surgery for endometriosis similar to our study [10].

The limitation of the present study is that it is an observational study, with no randomization, and we did not do a second look laparoscopy. Thus, further prospective randomized control trial studies are needed to validate our results.

\section{Conclusion}

Different techniques for haemostasis and ovarian reconstruction following cystectomy are use of bipolar coagulation, sutures, fibrin sealants and direct pressure. We reconstructed the ovaries by approximating the ovarian surface with a bowel grasper for few minutes, and it was associated with a successful outcome. Though this is a simpler technique, further welldesigned randomized controlled trials are needed.

Conflict of interest The authors, Dr. Paul P G, Dr. Harneeth Kaur, Dr. Dhivya Narasimhan, Dr. Gaurav Chopade and Dr. Dimple Kandhari declare that they have no conflict of interest. The authors alone are responsible for the content and writing of the paper.

Informed consent All procedures followed were in accordance with the ethical standards of the responsible committee on human experimentation (institutional and national) and with the Helsinki declaration of 1975, as revised in 2000(5). Informed consent was obtained from all patients for being included in the study.

\section{References}

1. Loh FH, Tan AT, Kumar J, Ng SC (1999) Ovarian response after laparoscopic ovarian cystectomy for endometriotic cysts in 132 monitored cycles. Fertil Steril 72:316-321

2. Valle RF, Sciarra JJ (2003) Endometriosis: treatment strategies. Ann N Y Acad Sci 997:229-239. doi:10.1196/annals.1290.026

3. Brosens IA, Van Ballaer P, Puttemans P, Deprest J (1996) Reconstruction of the ovary containing large endometriomas by an extraovarian endosurgical technique. Fertil Steril 66(4):517-521

4. Beretta P, Franchi M, Ghezzi F, Busacca M, Zupi E, Bolis P (1998) Randomized clinical trial of two laparoscopic treatments of endometriomas: cystectomy versus drainage and coagulation. Fertil Steril 70:1176-1180

5. Canis M, Mage G, Wattiez A, Chapron C, Pouly JL, Bassil S (1992) Second look laparoscopy after laparoscopic cystectomy of large ovarian endometriomas. Fertil Steril 58:611-619

6. Muzii L, Bellati F, Palaia I, Plotti F, Manci N, Zullo MA et al (2005) Laparoscopic stripping of endometriomas: a randomized trial on different surgical techniques. Part I: clinical results. Hum Reprod 20:1981-1986

7. Pellicano M, Bramante S, Guida M, Bifulco G, Di Spiezio SA, Cirillo D, Nappi C (2008) Ovarian endometrioma: postoperative adhesions following bipolar coagulation and suture. Fertil Steril 89(4):796-799

8. Ebert AD, Hollauer A, Fuhr N, Langolf O, Papadopoulos T (2009) Laparoscopic ovarian cystectomy without bipolar coagulation or sutures using a gelantine-thrombin matrix sealant (FloSeal): first support of a promising technique. Arch Gynecol Obstet 280:161-165

9. (1997) American, Society for Reproductive Medicine Revised classification of endometriosis: 1996. Fertil Steril 67:817-21

10. Vercellini P, Somigliana E, Viganò P, De Matteis S, Barbara G, Fedele L (2009) The effect of second-line surgery on reproductive performance of women with recurrent endometriosis: a systematic review. Acta Obstet Gynecol Scand 88(10):1074-1082

11. Yeung PP, Shwayder J, Pasic RP (2009) Laparoscopic management of endometriosis: comprehensive review of best evidence. J Minim Invasive Gynecol 16(3):269-281

12. Takeuchi H, Awaji M, Hashimoto M, Nakano Y, Mitsuhashi N, Kuwabara Y (1996) Reduction of adhesions with fibrin glue after laparoscopic excision of large ovarian endometriomas. J Am Assoc Gynecol Laparosc 3(4):575-579

13. Fedele L, Bianchi S, Zanconato G, Bergamini V, Berlanda N (2004) Bipolar electrocoagulation versus suture of solitary ovary after laparoscopic excision of ovarian endometriomas. J Am Assoc Gynecol Laparose 11(3):344-347

14. Dubuisson JB, Fauconnier A, Chapron C, Kreiker G, Nörgaard C (1998) Second look after laparoscopic myomectomy. Hum Reprod 13(8):2102-2106

15. Saeki A, Matsumoto T, Ikuma K, Tanase Y, Inaba F, Oku H, Kuno A (2010) The vasopressin injection technique for laparoscopic excision of ovarian endometrioma: a technique to reduce the use of coagulation. J Minim Invasive Gynecol 17(2):176-179

16. Pursifull NF, Morey AF (2007) Tissue glues and nonsuturing techniques. Curr Opin Urol 17(6):396-401 